\title{
Perfil audiológico de crianças com distúrbio primário de linguagem
}

\author{
Audiological profile in children with \\ primary language disorder \\ Perfil audiológico de niños con
trastorno del lenguaje primario
}
Eduarda Anzolin Pereira1 ${ }^{1}$, Laura Faustino Gonçalves², Patrícia Haas³ Karina Mary De Paiva ${ }^{4}$, Ana Paula Blanco-Dutra ${ }^{5}$

\begin{abstract}
1.Graduanda de fonoaudiologia da Universidade Federal de Santa Catarina - UFSC; Departamento de Fonoaudiologia. Florianópolis-SC, Brasil. ORCID: https://orcid.org/0000-0001-9251-0806

2.Graduanda de fonoaudiologia da Universidade Federal de Santa Catarina - UFSC; Departamento de Fonoaudiologia. Florianópolis-SC, Brasil. ORCID: https://orcid.org/0000-0002-0043-4349

3.Professora Doutora do Curso de Fonoaudiologia pela Universidade Federal de Santa Catarina - UFSC; Departamento de Fonoaudiologia. Florianópolis-SC, Brasil. ORCID: https://orcid.org/0000-0001-97977755

4.Professora Doutora do Curso de Fonoaudiologia pela Universidade Federal de Santa Catarina - UFSC; Departamento de Fonoaudiologia. Florianópolis-SC, Brasil. ORCID: https://orcid.org/0000-0001-7086$534 X$

5.Professora Doutora do Curso de Fonoaudiologia pela Universidade Federal de Santa Catarina - UFSC; Departamento de Fonoaudiologia. Florianópolis-SC, Brasil. ORCID: https://orcid.org/0000-0002-6495$\underline{7480}$
\end{abstract}

\section{Resumo}

Objetivo. Verificar evidências científicas do perfil audiológico de crianças com distúrbio primário de linguagem. Método. A busca por artigos científicos foi conduzida por dois pesquisadores independentes nas bases de dados Medline (Pubmed), LILACS, SciELO, Scopus, WEB OF SCIENCE e BIREME sem restrição de idioma, período e localização. Para complementar e evitar viés de risco foi realizada uma busca por literatura cinza no Google Scholar. Resultados. Do grupo de pacientes (1524 crianças), 62,87\% apresentaram comprometimento de linguagem primária. Houve associação significante entre comprometimento primário de linguagem e perfil audiológico alterado. Indivíduos com perfil audiológico atípico tiveram 63\% mais chance de apresentar comprometimento primário de linguagem quando comparado àqueles com perfil audiológico normal. Conclusão. É imprescindível desenvolver novas pesquisas que versem sobre programas de prevenção e promoção de saúde. Torna-se fundamental alertar e informar pais e responsáveis da importância de se tratar possíveis comprometimentos condutivos, os quais podem aparecer na infância, assim como atentá-los para os sinais de alerta que os filhos podem apresentar e que demonstram dificuldades de desenvolvimento de fala e linguagem.

Unitermos. Audição; Vias Auditivas; Percepção da Fala; Transtorno Específico de Linguagem; Crianças

\footnotetext{
Abstract

Objective. To verify scientific evidence of the audiological profile of children with primary language disorder. Method. The search for scientific articles was conducted by two independent researchers in the databases Medline (Pubmed), LILACS, SciELO, Scopus, WEB OF SCIENCE and BIREME without restriction of language, period, and location. To complement and avoid risk bias, a search for gray literature was carried out on Google Scholar. Results. From the group of patients (1524 children), $62.87 \%$ had impaired primary language. There was a significant association between primary language impairment and altered audiological profile. Individuals with atypical audiological profile were $63 \%$ more likely to have primary language impairment when compared to those with normal audiological profile. Conclusion. It is essential to develop new research that deals with prevention and health promotion
} 
programs. It is essential to alert and inform parents and guardians of the importance of treating possible conductive impairments, which may appear in childhood, as well as paying attention to the warning signs that their children may present and that demonstrate difficulties in the development of speech and language.

Keywords. Hearing; Auditory Pathways; Speech Perception; Specific Language Disorder; Children

\section{RESUMEN}

Objetivo. Verificar la evidencia científica del perfil audiológico de niños con trastorno del lenguaje primario. Método. La búsqueda de artículos científicos fue realizada por dos investigadores independientes en las bases de datos Medline (Pubmed), LILACS, SciELO, Scopus, WEB OF SCIENCE y BIREME sin restricción de idioma, período y ubicación. Para complementar y evitar el sesgo de riesgo, se realizó una búsqueda de literatura gris en Google Scholar. Resultados. Del grupo de pacientes ( $n=1524$ niños), 62,87\% tenían deterioro del lenguaje primario. Hubo una asociación significante entre el deterioro del lenguaje primario y el perfil audiológico alterado. Los individuos con un perfil audiológico atípico tenían un 63\% más de probabilidades de tener un deterioro del lenguaje primario en comparación con aquellos con un perfil audiológico normal. Conclusión. Es fundamental desarrollar nuevas investigaciones que se ocupen de los programas de prevención y promoción de la salud. Es fundamental alertar e informar a los padres y tutores sobre la importancia de tratar las posibles deficiencias conductivas, que pueden surgir en la infancia, así como prestar atención a las señales de alerta que puedan presentar sus hijos y que demuestren dificultades en el desarrollo del habla y el lenguaje.

Palabras clave: Audición; Vías auditivas; Percepción del habla; Trastorno específico del lenguaje; Niños

Trabalho realizado na Universidade Federal de Santa Catarina - UFSC; Departamento de Fonoaudiologia. Florianópolis-SC, Brasil.

\section{INTRODUÇÃO}

A integralidade do sistema auditivo, ou seja, limiares auditivos dentro dos padrões de normalidade e funcionamento adequado das estruturas centrais, são prérequisito fundamental para a aquisição e desenvolvimento da linguagem oral. Nos primeiros anos de vida, as experiências auditivas são fundamentais para a organização cortical, a qual garante o desenvolvimento normal da audição e da linguagem, já que a percepção e desenvolvimento da fala são obtidas por meio da audição ${ }^{1}$. O desenvolvimento das habilidades de linguagem e fala necessitam da integridade e maturação neuromuscular e do 
sistema sensorial, contribuindo para o desenvolvimento de um padrão articulatório de um idioma. As perdas auditivas permanentes ou temporárias, como as causadas por otite média, podem resultar em importantes dificuldades para a aquisição e desenvolvimento da linguagem e fala².

A perda auditiva leve, mesmo que unilateral, cuja causa mais comum na primeira infância são as otites médias, leva à percepção de estímulos sonoros distorcidos ${ }^{3}$. desenvolvimento de uma perda auditiva condutiva, nos três primeiros anos de vida, mesmo que de grau leve representa um fator de risco à aquisição de um idioma, tanto pela dificuldade na percepção de sons da fala, como as consoantes surdas ou fricativas /s/ e / $/$ /, quanto pelos erros fonéticos na pronúncia dos fonemas / $/ /$ e / $/$. Assim, $O$ tratamento precoce das otites médias nesta faixa etária é considerado essencial.

A American Speech, Language and Hearing Association $(\mathrm{ASHA})^{4}$ define como uma desordem na comunicação as dificuldades na recepção e/ou no processamento de um sistema simbólico que podem ser observados em nível da audição, linguagem e/ou fala, podendo variar quanto a severidade e serem de origem desenvolvimental ou adquirida. Podem ser distúrbios primários, que têm sua manifestação primária ou idiopática ou secundário, resultante de um distúrbio maior, isolado ou concomitantemente a outros. Dentre as alterações primárias de linguagem destacam-se as desordens na articulação, como o comprometimento no sistema de sons da língua e 
suas regras que resultam em uma emissão oral anormal de um ou mais fonemas; desordens na aquisição e desenvolvimento da linguagem oral que comprometem a aquisição, compreensão e expressão; e, por fim, desordens na aquisição e desenvolvimento da linguagem escrita.

Os processos de aprendizagem e da linguagem são bastante complexos, envolvem redes de neurônios distribuídas por diversas regiões cerebrais e se relacionam à percepção da fala, sendo dependentes da integridade das vias auditivas, tanto periférica quanto central ${ }^{5}$.

No período de aquisição fonológica é esperado que haja substituições e/ou omissões de fonemas, desde a não realização de segmentos até a não produção de estruturas silábicas complexas ${ }^{6}$. Durante esse período, o domínio do sistema fonológico da língua é espontâneo, em uma determinada sequência e faixa etária, que vai dos quatro aos seis anos de idade. No entanto, para algumas crianças isso não acontece de forma espontânea, ou seja, elas apresentam dificuldade de organização cognitiva, de estabelecimento do sistema fonológico da língua e de adaptação à informação oral recebida. Assim, a fala caracteriza-se por omissões e substituições de fonemas, originando o desvio fonológico², o qual não possui fatores orgânicos identificáveis como anormalidades anatômicas, funcionais e/ou deficiência auditiva7. Embora alguns autores apontem fatores que influenciam o desvio fonológico, sua etiologia ainda é desconhecida². 
A dificuldade na aquisição e/ou no desenvolvimento da linguagem escrita ocorre em indivíduos que apresentam dificuldade tanto de decodificação fonológica como de compreensão da linguagem oral e/ou escrita. Embora essa manifestação seja evidente durante o aprendizado da leitura e da escrita, alguns sinais que expressam dificuldade mais ampla na linguagem podem ser observados nos anos préescolares, como vocabulário pobre, uso inadequado da gramática e problemas no processamento fonológico. Nas séries iniciais, além das dificuldades de reconhecimento e compreensão em leitura, podem aparecer problemas de compreensão auditiva e discurso ${ }^{5}$.

Estudos apontam para uma relação entre os distúrbios da comunicação oral associados ao desenvolvimento educacional e social, e muitos dos fatores envolvidos podem ser evitáveis se houver a detecção precoce. Porém, o interesse nesta deteç̧ão e tratamento da comunicação oral e de distúrbios auditivos está apenas começando, e é de suma importância que pais e cuidadores estejam mais conscientes dessas dificuldades para que possam ser abordados nas configurações públicas de atenção primária ${ }^{8,9}$.

Tendo isto em vista, há maior probabilidade de aprimoramento de linguagem expressiva e receptiva, alfabetização, desenvolvimento social, emocional e acadêmico com a deteç̧ão precoce ${ }^{10}$.

Frente ao exposto, este estudo tem como objetivo principal caracterizar, por meio de uma revisão sistemática da literatura, organizada no formato PICOS, o perfil 
audiológico de crianças com distúrbios primários de linguagem, visando responder a seguinte pergunta norteadora de pesquisa: qual o perfil audiológico de crianças com distúrbios primários de linguagem?

\section{MÉTODO}

Os descritores foram selecionados a partir dos vocabulários controlados Descritores em Ciências da Saúde (DeCS) e Medical Subject Heading Terms (MeSH), haja vista a sua ampla utilização pela comunidade científica para a indexação de artigos na base de dados PubMed. Utilizou-se como estratégia de busca a combinação de descritor e operador booleano: (audiologic profile) and (children) and (phonological disorder) and (phonological disorder). As buscas por artigos científicos foram conduzidas por dois pesquisadores independentes nas bases de dados eletrônicas MEDLINE (Pubmed), LILACS, SciELO, SCOPUS, WEB OF SCIENCE e BIREME, sem restrição de idioma, período e localização. Para complementar e evitar viés de risco foi realizada uma busca por literatura cinza no Google Scholar.

A presente revisão sistemática foi conduzida conforme as recomendações PRISMA (Preferred Reporting Items for Systematic reviews and Meta-Analyses) ${ }^{11}$, visando obter 0 critério mais rigoroso de protocolo de evidência científica. A pesquisa foi estruturada e organizada na forma PICOS, que representa um acrônimo para População alvo, a Intervenção, Comparação e "Outcomes" (desfechos). População de interesse ou problema de saúde (P) 
corresponde a crianças; intervenção (I): terapia fonoaudiológica; comparação (C): perfil audiológico; outcome (O): transtorno específico de linguagem transtorno fonológico, e dificuldade de leitura e escrita; (S): estudo transversal, estudo observacional, relatos de caso, estudos de caso-controle, ensaios clínicos controlados, estudos de coorte (Tabela 1).

Tabela 1. Descrição dos componentes do PICOS.

\begin{tabular}{c|l}
\hline Acrônimo & Definição \\
\hline $\mathbf{P}$ & $\begin{array}{l}\text { Crianças com distúrbio primário de } \\
\text { linguagem }\end{array}$ \\
\hline $\mathbf{C}$ & Terapia fonoaudiológica \\
\hline $\mathbf{0}$ & Perfil Audiológico \\
\hline $\mathbf{S}$ & $\begin{array}{l}\text { Transtorno específico de linguagem } \\
\text { Transtorno fonológico } \\
\text { Dificuldade de leitura e escrita }\end{array}$ \\
\hline & $\begin{array}{l}\text { Estudo transversal } \\
\text { Estudo observacional } \\
\text { Estudos de caso-controle } \\
\text { Ensaios clínicos controlados } \\
\text { Estudo de coorte } \\
\text { Relatos de caso } \\
\text { Estudos de Intervenção. }\end{array}$ \\
\hline
\end{tabular}

\section{Critérios de seleção}

Foram incluídos estudos sem restrição de idioma, período e localização. A Tabela 2 representa os critérios de inclusão e exclusão aplicados especificamente para esta pesquisa. O estudo obteve pontuação 12 no protocolo modificado de Pithon et al. ${ }^{9}$ para avaliação da qualidade dos mesmos. 
Foram excluídos estudos publicados nos formatos de Cartas ao editor, diretrizes, revisões de literatura, revisões narrativas, revisões sistemáticas, meta análises e resumos. Estudos indisponíveis na íntegra, também foram excluídos (Tabela 2).

Tabela 2. Síntese dos critérios de incluso/exclusão.

\begin{tabular}{cc}
\hline Critérios de Inclusão \\
\hline Delineamento & $\begin{array}{c}\text { Relatos de casos } \\
\text { Estudos de casos e controle } \\
\text { Ensaios clínicos controlados } \\
\text { Estudos de coorte } \\
\text { Estudos em triagem } \\
\text { Estudos observacionais }\end{array}$ \\
\hline Localização & Sem Restrição \\
\hline Idioma & Sem restrição \\
\hline Período & Sem restrição \\
\hline Delineamento & Critérios de Exclusão \\
& Cartas ao editor \\
& Diretrizes \\
& Revisões de literatura \\
& Revisões sistemáticas \\
& Meta-análises \\
\hline Estudos & Estudos pouco claros \\
& Mal descritos ou inadequados \\
\hline
\end{tabular}

\section{Análise dos dados}

A extração dos dados para o processo de elegibilidade dos estudos foi realizada utilizando-se uma ficha própria para revisão sistemática elaborada por dois pesquisadores em Programa Excel ${ }^{\circledR}$, na qual os dados extraídos foram adicionados por um dos pesquisadores e, então, conferidos 
por outro pesquisador. Inicialmente foram selecionados de acordo com o título; em seguida, os resumos foram analisados e apenas os que fossem potencialmente elegíveis foram selecionados. Com base nos resumos, artigos foram selecionados para leitura integral, foram admitidos os que atendiam a todos os critérios pré-determinados.

A qualidade dos métodos utilizados no estudo foi avaliada pelos revisores de forma independente $(\mathrm{PH}, \mathrm{APBD}$, KMP), de acordo com a recomendação do protocolo PRISMA $^{12}$. A avaliação priorizou a descrição clara das informações. Neste ponto, a revisão foi realizada às cegas, mascarando os nomes dos autores e revistas, evitando qualquer viés potencial e conflito de interesses.

Inicialmente os revisores de elegibilidade (PH, APBD, KMP) foram calibrados para a realização da revisão sistemática por LFG e EAP. Após a calibração e esclarecimentos de dúvidas, os títulos e resumos foram examinados por um revisor de elegibilidade $(\mathrm{PH})$, de forma independente, os quais não estavam cegos para o nome dos autores e das revistas.

\section{RESULTADOS E DISCUSSÃO}

Os resultados obtidos nesta pesquisa e observados na Figura 1 destacam que foram realizadas as exclusões por duplicidade, título, resumo e leitura completa. Ao final do processo de seleção, um estudo adequou-se a todos os critérios de elegibilidade. É possível verificar toda a seleção de admissibilidade dos artigos por meio da Figura 1 . O estudo 
incluído nesta pesquisa é do tipo observacional transversal retrospectivo. Para os dados obtidos dos estudos elegíveis, estes também foram transportados para uma planilha no mesmo programa, a fim de organizar os resultados como demonstrado na Tabela 3.

Figura 1. Fluxograma do processo de busca.

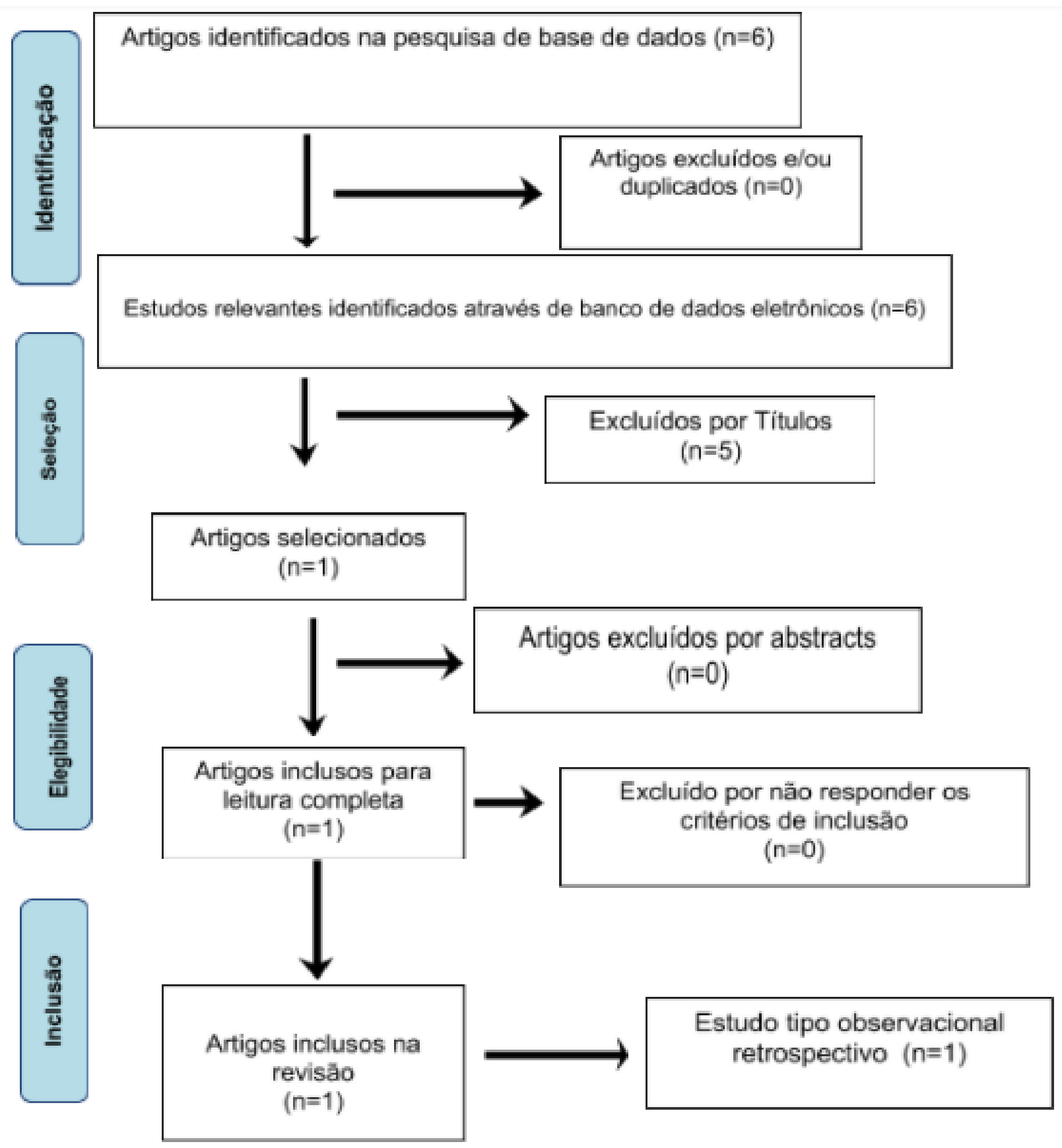


Tabela 3. Classificação das referências obtidas nas bases de dados Pubmed, Scielo, Lilacs, Web Of Science e Scopus.

\begin{tabular}{|c|c|c|c|c|c|}
\hline Descritores & No & $\begin{array}{c}\text { Referências } \\
\text { excluídas }\end{array}$ & Motivo & Selecionado & $\begin{array}{c}\text { Banco de } \\
\text { dados }\end{array}$ \\
\hline $\begin{array}{l}\text { (audiologic profile) and } \\
\text { (children) and (phonological } \\
\text { disorder) and (phonological } \\
\text { disorder) }\end{array}$ & 5 & 4 & $\begin{array}{l}\text { Excluídos por } \\
\text { título (4) }\end{array}$ & 1 & Pubmed \\
\hline $\begin{array}{l}\text { (audiologic profile) and } \\
\text { (children) and (phonological } \\
\text { disorder) and (phonological } \\
\text { disorder) }\end{array}$ & - & - & - & - & Lilacs \\
\hline $\begin{array}{l}\text { (audiologic profile) and } \\
\text { (children) and (phonological } \\
\text { disorder) and (phonological } \\
\text { disorder) }\end{array}$ & 1 & 1 & $\begin{array}{l}\text { Excluído por } \\
\text { título (1) }\end{array}$ & 1 & Scielo \\
\hline $\begin{array}{l}\text { (audiologic profile) and } \\
\text { (children) and (phonological } \\
\text { disorder) and (phonological } \\
\text { disorder) }\end{array}$ & - & - & - & - & $\begin{array}{l}\text { WEB OF } \\
\text { SCIENCE }\end{array}$ \\
\hline $\begin{array}{l}\text { (audiologic profile) and } \\
\text { (children) and (phonological } \\
\text { disorder) and (phonological } \\
\text { disorder) }\end{array}$ & - & - & - & - & Bireme \\
\hline $\begin{array}{l}\text { (audiologic profile) and } \\
\text { (children) and (phonological } \\
\text { disorder) and (phonological } \\
\text { disorder) }\end{array}$ & - & - & - & - & SCOPUS \\
\hline Total & 6 & 5 & & 1 & Pubmed \\
\hline
\end{tabular}

O estudo admitido nesta pesquisa realizou análise dos prontuários do Serviço de Fonoaudiologia e Pediatria de um Centro de Saúde ${ }^{13}$. Foram coletados os dados de diagnóstico fonoaudiológico de todos os pacientes submetidos à avaliação fonoaudiológica completa entre os anos de 1985 e 2009. Todos os sujeitos eram falantes nativos do português brasileiro, destes 2424 foram convidadas para participarem 
do grupo de estudo (caso) e 186 crianças pertenceram ao grupo controle. O grupo controle teve a mesma faixa etária do grupo de estudo; as crianças procuraram o mesmo centro de saúde e não apresentavam queixas ou antecedentes de distúrbio de linguagem.

Em relação à avaliação fonoaudiológica do grupo de estudo, foram consideradas as variáveis sexo, idade, diagnóstico fonoaudiológico de distúrbio primário de linguagem, incluindo transtorno específico de linguagem, desvio fonológico e déficit na leitura e escrita. O diagnóstico primário, em caso de comorbidades, foi determinado considerando o diagnóstico mais grave, óbvio e dominante, de acordo com a avaliação inicial da criança no serviço. Para a avaliação audiológica, as variáveis consideradas foram a imitanciometria (timpanograma e reflexos acústicos) e audiometria tonal. Essas variáveis foram classificadas de acordo com os seguintes critérios; audiometria tonal dentro da normalidade (limiares tonais até $15 \mathrm{~dB}$ NA) e perda auditiva condutiva, perda auditiva mista, perda auditiva isolada (em uma ou duas frequências) ou não testada. Ressalta-se que a perda auditiva neurossensorial foi excluída porque esse tipo de diagnóstico auditivo não se enquadra nos casos de distúrbio primário de linguagem, uma vez que o distúrbio de linguagem seria considerado secundário, portanto, classificado como outros diagnósticos ${ }^{13}$. 


\section{Alterações Auditivas e de Linguagem}

Para verificar a associação entre deficiência auditiva e alteração de linguagem, foi criada uma variável que representa o perfil audiológico, formada pelo somatório das variáveis que foram incluídas na avaliação audiológica (audiometria tonal liminar e imitanciometria). Essa variável foi dicotomizada em duas categorias como perfil audiológico normal ou alterado. Para a classificação 'perfil audiológico normal', tanto a imitanciometria quanto a audiometria tonal deveriam ser normais em ambas as orelhas; para a classificação 'perfil audiológico alterado' foi considerada a presença de resultados anormais em uma ou nas duas avaliações, em uma ou nas duas orelhas ${ }^{13}$.

Como resultado, observou-se elevada prevalência de comprometimento de linguagem primário $(62,87 \%)$ na amostra, representando 1524 indivíduos. Do total de sujeitos com comprometimento de linguagem primário, 477 participantes não possuíam registro de avaliação audiológica. Entre os indivíduos com comprometimento de linguagem primário e dados de avaliação audiológica $(n=1047)$, os comprometimentos estavam presentes em 616 indivíduos $(58,84 \%)$ com desvio fonológico; 322 sujeitos $(30,75 \%)$ transtorno específico de linguagem; e 109 indivíduos $(10,41 \%)$ deficit na leitura e na escrita ${ }^{13}$.

Em relação ao perfil audiológico, 911 indivíduos $(87,01 \%)$ completaram a audiometria tonal liminar. Diante disso, predominaram os limiares auditivos dentro da normalidade $(81,34 \%)$. Em relação ao timpanograma, foram 
obtidos dados de 1035 indivíduos (98,85\%). com prevalência da curva do tipo A $(56,24 \%)$. Em relação aos reflexos acústicos, foram obtidos dados de 946 crianças $(90,3 \%)$, sendo que para esses indivíduos, a presença de reflexos acústicos foi observada em $51,7 \%{ }^{13}$.

Para analisar a associação entre apresentar comprometimento de linguagem primário e perfil audiológico alterado, foram analisadas crianças com comprometimento de linguagem primário $(n=1047)$ e crianças sem queixas de distúrbios de linguagem, grupo controle $(n=186)$. Observouse associação significante entre perfil audiológico alterado e comprometimento do desenvolvimento da linguagem, desvio fonológico e déficit de leitura e escrita. Destaca-se que indivíduos que apresentavam perfil audiológico atípico tinham 63\% mais chances de apresentar algum comprometimento de linguagem primária em comparação aos que apresentavam perfil audiológico normal (razão de risco 1,63$)^{13}$.

A maioria dos indivíduos com comprometimento de linguagem primário e que realizaram audiometria tonal liminar, apresentaram resultados dentro dos padrões da normalidade, seguidos de perda auditiva condutiva em $15,47 \%$ dos casos e perda auditiva mista ou isolada em 3,19\%. A ocorrência verificada em perda auditiva mista e isolada (geralmente leve e em altas frequências) também pode ter relação com distúrbios condutores. Os autores também indicam que na otite média, a disseminação de toxinas da orelha média para a cóclea pode ocorrer através 
da janela redonda, o que levaria à perda auditiva neurossensorial permanente ou temporária ${ }^{13}$.

\section{Achados dos exames audiológicos}

Com relação a timpanometria, a curva do tipo $A$, considerada normal, foi verificada como predominante. Observou-se uma ocorrência de curvas timpanométricas do tipo B $(21,84 \%)$ e tipo C $(18,16 \%)$. Os outros tipos de timpanogramas ( $\mathrm{Ar}$, Ad e pico duplo) foram inferiores a $5 \%$. A alta ocorrência de timpanogramas anormais do tipo (B e C) sugerem uma possível detecção de alterações precoces e/ou subclínicas pela imitanciometria, que não foram encontradas pela audiometria tonal liminar. Este fator, explicaria um maior número de alterações na timpanometria em relação aos resultados da audiometria tonal. É importante ressaltar que mais indivíduos apresentaram resultados na imitanciometria em comparação com a audiometria tonal, dado que a primeira se trata de uma avaliação objetiva; por isso, muitas crianças que não realizaram a audiometria tonal foram avaliadas apenas pela imitanciometria ${ }^{13}$.

Sobre os reflexos acústicos, os resultados obtiveram condições normais em $(51,7 \%)$ dos casos, com presença de reflexos acústicos em todas as frequências testadas. Entretanto, muitos indivíduos apresentaram reflexos acústicos ausentes $(27,16 \%)$ em todas as frequências testadas, e $21,14 \%$ ausentes apenas em uma ou duas frequências ${ }^{13}$. 


\section{Características demográficas e Alterações de}

\section{Linguagem}

Com relação às características demográficas, encontraram associação estatisticamente significativa entre pertencer à faixa etária mais elevada ( 7 a 12 anos) e apresentar desvio fonológico. O que pode ser explicado pelo fato de o desenvolvimento fonológico no português brasileiro ocorrer até os sete anos de idade. Consequentemente, em vista disto, a fala e/ou linguagem da criança é considerada, muitas vezes, adequada por familiares e profissionais da educação e da saúde, ainda que tenha a presença de processos fonológicos e distorções/desvios fonéticos, sejam eles esperados para a idade ou não. Em relação ao transtorno específico de linguagem, a faixa etária predominante foi a mais jovem (até os 6 anos), o que foi considerado como esperado por conta do diagnóstico de transtorno específico de linguagem ser confirmado por volta de quatro ou cinco anos de idade. Por último, foi encontrada relação entre a faixa etária de ( 7 a 12 anos) e apresentar dificuldade de leitura e escrita, o que pode ser justificado pela idade em que se inicia a alfabetização formal no Brasil, aos sete anos, no primeiro ano do ensino fundamental. Logo, os distúrbios de leitura e escrita não foram detectados anteriormente a essa idade ${ }^{13}$.

A aquisição e o desenvolvimento da linguagem não consistem em um processo único, pois sofrem interferência de diversos fatores associados ${ }^{14}$. Os distúrbios de causa idiopática referentes ao desenvolvimento da linguagem oral 
e/ou escrita, que acometem crianças e adolescentes não ocorrem em conjunto com outras anormalidades, tais como: deficiência mental, paralisia cerebral, deficiências auditivas e outras. Estes distúrbios, idiopáticos ou secundários podem ser aumentados por influências externas, como por exemplo, diferenças culturais, orientação insuficiente ou inapropriada ${ }^{15}$.

Associações significantes foram encontradas entre pertencer à faixa etária mais elevada $(7-12$ anos $p 0,026)$ e apresentar desvio fonológico, assim como pertencer a faixa etária mais jovem (até os 6 anos; $p<0,001$ ) e possuir transtorno específico de linguagem, e, por fim, possuir entre 7 e 12 anos de idade $(p<0,001)$ e apresentar dificuldade de leitura e escrita ${ }^{13}$.

$\mathrm{Na}$ maioria das vezes, estudos epidemiológicos sobre distúrbios da comunicação apontam valores de prevalência e incidência quanto à idade, sexo, nível socioeconômico e diagnóstico do distúrbio da comunicação. Tem-se observado maior prevalência de distúrbios idiopáticos da comunicação no sexo masculino e a partir dos três anos, que se estendem até os oito anos de idade, sendo a fase crítica o período de quatro a seis anos, com decréscimo a partir de então. Sobre os fatores socioeconômicos, estudos apontam que pais com baixo nível educacional têm mais chances de ter filhos com dificuldades de linguagem, além de problemas para observar e/ou relatar tais dificuldades ${ }^{15}$. 
A maioria das crianças com comprometimento de linguagem primário eram do sexo masculino $(64,19 \%)$ e pertenciam à faixa etária até 6 anos $(67,15 \%)^{13}$.

Crianças do sexo masculino são predominantemente diagnosticadas com transtorno específico de linguagem ${ }^{16}$. Fato que pode ser indicado, pois o cérebro dos meninos apresenta uma maturação mais lenta quando comparado ao cérebro das meninas, e por isso, tornam-se mais expostos a estes e outros riscos. Outros fatores, como a genética, podem também estar associados como importantes determinantes na prevalência de distúrbios específicos de linguagem e de dislexia na população masculina. Além disso, foi constatado que a demanda por intervenção fonoaudiológica foi predominantemente do sexo masculino, sugerindo que a aquisição e desenvolvimento da linguagem da linguagem entre meninos e meninas acontecem de forma diferente, em razão da forma como a criança interage com o meio, configuração de criação ou pelo fator maturacional do cérebro ser normalmente mais lento nos meninos ${ }^{17}$.

Verificou-se que $15,47 \%$ dos indivíduos apresentaram perda auditiva condutiva, e perda mista ou isolada em 3,19\% dos casos (geralmente leve e em altas frequências), o que pode ser relacionado também com distúrbios condutores ${ }^{13}$. Em outros estudos também foi possível observar que as alterações encontradas foram em sua maioria de origem condutiva, seguidas por alterações sensorioneurais e mistas $^{18,19}$. 
Alterações na via auditiva impactam diretamente no processo de aquisição e desenvolvimento da linguagem, as perdas condutivas levam a uma redução na intensidade dos sons que chegam à cóclea, resultantes da diminuição na mobilidade da membrana timpânica, a percepção passa a ser reduzida, abafada e com perda de profundidade, evidenciando que esta condição de audição pode gerar grande impacto na linguagem ${ }^{20,21}$. Cerca de $80 \%$ das crianças têm pelo menos um episódio de otite média secretora (OMS) até os oito anos de idade. Destas, aproximadamente $55 \%$ possuem perda auditiva leve nas frequências da fala. As perdas auditivas flutuantes na otite média (que alternam períodos de audição normal) convertem-se em uma estimulação sonora inconsistente do sistema auditivo central, prejudicando a percepção dos sons da fala. Além disso, o fluido na orelha média, decorrente da otite média, pode gerar ruído junto à cóclea, contribuindo ainda mais para dificuldades na percepção sonora pela criança. Em casos de otite média secretora ou crônica, a perda auditiva condutiva também pode coexistir com uma perda auditiva sensorioneural, ou seja, perda auditiva mista em altas frequências ${ }^{22}$. A perda auditiva por OMS pode gerar um prejuízo de 15 a 40dBNA na orelha acometida ${ }^{23}$. Isto acarretará numa diminuição da discriminação de fala pela consequente perda na acuidade auditiva, sendo assim, a duração deste quadro clínico pode causar danos cognitivos ao indivíduo, no que diz respeito a articulação da fala ou na aquisição da linguagem. 
A privação sensorial resultante de uma secreção na orelha média, acentuada pelas repetições e duração destes episódios, é capaz de impactar na percepção da fala e prejudicar a compreensão, especialmente em ambientes ruidosos. Estes fatores refletem de forma global na vida da criança, nas áreas educacional, emocional, social, afetando o desenvolvimento da linguagem e processamento auditivo, já que o desenvolvimento da linguagem necessita do funcionamento do sistema auditivo periférico e central, para detectar, perceber, transmitir, memorizar e integrar experiências sonoras. Portanto, a detecção e a intervenção precoce nesta população, aumentam as chances de melhora na linguagem, aprendizagem e desenvolvimento socioemocional 24,25 .

Alguns comportamentos que podem ser observados em crianças com otite média podem sugerir uma privação auditiva, como virar a cabeça em direção à fonte sonora, pedidos frequentes de repetição, intensidade vocal elevada ou reduzida, leitura labial, desatenção, isolamento e dificuldade no aprendizado. Estas manifestações podem passar despercebidas por pais e educadores, prejudicando 0 desenvolvimento da linguagem e rendimento escolar ${ }^{26}$.

Em relação a timpanometria, os achados foram semelhantes aos encontrados por outros autores ${ }^{18}$ que encontraram, respectivamente, cerca de $45 \%$ e $35 \%$ de timpanogramas desviados (Tipos B, C, $\mathrm{Ar}$ ou $\mathrm{Ad})^{13}$. A timpanometria verifica a mobilidade do sistema tímpanoossicular em função da variação introduzida no meato 
acústico externo, mostrando-se eficiente no reconhecimento precoce das afecções de orelha média ${ }^{27}$. As timpanometrias com curva do tipo B ou C estão associadas com as perdas auditivas do tipo condutivas. Curvas do tipo B podem apresentar-se em casos de otite média com evolução, enquanto a curva do tipo $C$ é encontrada nos casos de involução da otite que ainda apresentam disfunção tubária ${ }^{28}$.

Para os reflexos acústicos, os resultados encontrados são semelhantes aos descritos em outro estudo ${ }^{24}$, que observou em média $70 \%$ da presença de reflexos acústicos em todas as frequências. É relevante ressaltar que a ausência de reflexos acústicos, especialmente em uma ou duas frequências, não representa indicativo de limiares auditivos anormais ${ }^{13}$. Em perdas auditivas condutivas, os reflexos acústicos encontram-se ausentes ou elevados pela reduzida transmissão do estímulo sonoro à orelha interna e redução na mobilidade das estruturas da orelha média, visto que limiares acústicos ausentes ou elevados ocorrem quando a resposta do reflexo não está presente ao nível esperado de 70 a $90 \mathrm{~dB}^{29}$, podendo explicar a incidência da ausência dos reflexos acústicos nestes indivíduos, se presente 0 comprometimento condutivo nos mesmos.

Estes resultados trazem uma importante base para as intervenções fonoaudiológicas no acompanhamento de crianças, visto que a chance de crianças que possuem distúrbios auditivos condutivos apresentarem distúrbios primários de linguagem é maior. Isso aumenta a importância da elaboração de ações de promoção e prevenção à saúde, 
assim como a criação de programas de intervenção, já que as perdas auditivas condutivas em crianças são recorrentes e podem causar dificuldades significativas no seu desenvolvimento ${ }^{13}$.

O profissional de saúde precisa ter um olhar ampliado e conhecer as ações de promoção, prevenção e recuperação da saúde fonoaudiológica da população ${ }^{30}$. Portanto, é preciso conhecimento sobre as condições de saúde de cada grupo através do delineamento da população usuária dos serviços coletivos mediante estudos epidemiológicos, com o intuito de elaborar políticas adequadas à população.

As informações referentes ao estudo admitido estão sintetizadas na Tabela 4.

\section{CONCLUSÃO}

Limiares auditivos dentro da normalidade foram constatados em $81,34 \%$ dos casos; a curva do tipo A configurou-se como a mais prevalente em 56,24\%; a presença de reflexos acústicos foi observada em 51,7\%. Pode-se concluir que o perfil audiológico de crianças com distúrbios primários de linguagem caracteriza-se predominantemente dentro dos padrões de normalidade. Porém, algumas crianças estudadas apresentam alterações na timpanometria e reflexos acústicos. Como o estudo sugere que crianças com perfil audiológico alterado podem ter $63 \%$ mais chances de apresentar comprometimento de linguagem, é imprescindível desenvolver novas pesquisas que versam sobre programas de prevenção e promoção de 
saúde. Torna-se fundamental alertar e informar pais e responsáveis da importância de se tratar possíveis comprometimentos condutivos, os quais podem aparecer na infância, assim como atentá-los para os sinais de alerta que os filhos podem apresentar e que demonstram dificuldades de desenvolvimento de fala e linguagem.

Tabela 4. Síntese do artigo incluído.

\begin{tabular}{|c|c|c|c|c|c|}
\hline $\begin{array}{c}\text { Autor/ } \\
\text { Ano/ Local } \\
\text { de } \\
\text { publicação }\end{array}$ & Objetivo & Amostra & Método & Resultados & Conclusão \\
\hline $\begin{array}{c}\begin{array}{c}\text { Pereira et } \\
\text { al., }\end{array} \\
2015^{13} \\
\text { Brasil }\end{array}$ & $\begin{array}{l}\text { Caracterizar as } \\
\text { crianças } \\
\text { falantes do } \\
\text { Português } \\
\text { Brasileiro } \\
\text { atendidas no } \\
\text { Serviço de } \\
\text { Fonoaudiologia } \\
\text { de um Centro } \\
\text { de Saúde, no } \\
\text { período de } 1985 \\
\text { a } 2009, \text { com } \\
\text { diagnóstico de } \\
\text { comprometimen } \\
\text { to primário de } \\
\text { linguagem } \\
\text { quanto ao perfil } \\
\text { demográfico e } \\
\text { audiológico }\end{array}$ & $\begin{array}{c}2.610 \\
(n=2424 \\
\text { grupo estudo) } \\
(n=186 \text { grupo } \\
\text { controle) }\end{array}$ & $\begin{array}{l}\text { Foi realizado } \\
\text { levantamento de } \\
\text { prontuários, com } \\
\text { coleta de dados } \\
\text { diagnósticos } \\
\text { audiológicos e de } \\
\text { linguagem, totalizando } \\
2.424 \text { indivíduos no } \\
\text { grupo de estudo. Além } \\
\text { disso, foram coletados } \\
\text { dados de avaliação } \\
\text { audiológica de } 186 \\
\text { crianças sem queixas } \\
\text { de distúrbios de } \\
\text { linguagem para que } \\
\text { constituíssem o grupo } \\
\text { controle. }\end{array}$ & $\begin{array}{l}\text { Do grupo de estudo, } 1524 \\
\text { crianças (62,87\%) } \\
\text { apresentaram } \\
\text { comprometimento de } \\
\text { linguagem primário. } \\
\text { Foram observadas as } \\
\text { seguintes ocorrências; } \\
\text { desvio fonológico = } \\
58,84 \%, \\
\text { comprometimento do } \\
\text { desenvolvimento da } \\
\text { linguagem = } 30,75 \% \text { e } \\
\text { déficit de leitura e escrita } \\
=10,41 \%, \text { com } \\
\text { predomínio do sexo } \\
\text { masculino (64,19\%) e } \\
\text { faixa etária até } 6 \text { anos } \\
\text { (67,15\%). Para o perfil } \\
\text { audiológico, houve } \\
\text { predomínio dos limiares } \\
\text { auditivos normais } \\
\text { (81,34\%), seguidos da } \\
\text { perda auditiva condutiva } \\
\text { (15,47\%). Houve } \\
\text { associação significante } \\
\text { entre comprometimento } \\
\text { primário de linguagem e } \\
\text { perfil audiológico alterado. } \\
\text { Indivíduos com perfil } \\
\text { audiológico anormal } \\
\text { tiveram } 63 \% \text { mais chance } \\
\text { de apresentar } \\
\text { comprometimento } \\
\text { primário de linguagem do } \\
\text { que aqueles com perfil } \\
\text { audiológico normal. }\end{array}$ & $\begin{array}{c}\text { Sugere-se que ter um } \\
\text { perfil audiológico } \\
\text { anormal seria um } \\
\text { fator de risco para } \\
\text { comprometimento } \\
\text { primário de } \\
\text { linguagem. Assim, } \\
\text { esses achados podem } \\
\text { servir de base para o } \\
\text { monitoramento } \\
\text { auditivo e } \\
\text { fonoaudiológico } \\
\text { infantil, e uma } \\
\text { importante } \\
\text { ferramenta para o } \\
\text { planejamento de } \\
\text { ações de promoção e } \\
\text { prevenção da saúde, } \\
\text { bem como para o } \\
\text { desenvolvimento e } \\
\text { implementação de } \\
\text { programas de } \\
\text { intervenção. }\end{array}$ \\
\hline
\end{tabular}




\section{REFERÊNCIAS}

1.Costa LD, Quinto SMS, Didoné DD, Rechia IC, Garcia, MV, Biaggio EPV. Audição e linguagem em crianças nascidas a termo e pré-termo. Audiol Commun Res 2016;21:1-7. https://doi.org/10.1590/23176431-2016-1672

2.Caumo DT, Ferreira MIDC. Relação entre desvios fonológicos e processamento auditivo. Rev Soc Bras Fonoaudiol 2009;14:23440. https://doi.org/10.1590/S1516-80342009000200015

3. Hyppolito MA, Vieira L, Elias MV, Rodrigues DO, Bisanha AA, Manfredi AK. A audição e a linguagem da criança após tratamento da otite média crônica secretora com tubo de ventilação. Braz J Otorhinolaryngol 2007;73(suppl 2).

http://oldfiles.bjorl.org/triologico2007/anais/artigos/4059.htm

4.Andrade CRF. Prevalência das desordens idiopáticas da fala e da linguagem em crianças de um a onze anos de idade. Rev Saúde Pública. 1997;31:495-501.

89101997000600008

5. Machado CSS, Valle HLBS, Paula KM, Lima SS. Caracterização do processamento auditivo das crianças com distúrbio de leitura e escrita de 8 a 12 anos em tratamento no centro clínico de fonoaudiologia da Pontifícia Universidade Católica de Minas Gerais. Rev CEFAC 2011;13:504-12.

https://doi.org/10.1590/S1516-

18462010005000119

6.Ceron MI, Gubiani MB, Oliveira CR, Gubiane MB, Soares MK. Prevalence of phonological disorders and phonological processes in typical and atypical phonological development. CoDAS 2017;29:1-9. https://doi.org/10.1590/2317-1782/20172015306

7.Castro WL, Sousa CCA, Farias RRS. Relationship of auditory perception with speech language awareness of children with phonological deviation. Res Soc Develop 2021;10:1-14. https://doi.org/10.33448/rsd-v10i1.12020

8. Oliveira PS, Penna LM, Lemos SMA. Desenvolvimento da linguagem e deficiência auditiva: revisão de literatura. Rev CEFAC 2015;17:2044-55. https://doi.org/10.1590/1982-0216201517611214 9.Goulart BNG, Chiari BM, Almeida CPB. Factors associated with speech, hearing and language disorders among children in a primary care outpatient center. J Hum Growth Dev 2017;27:281. http://dx.doi.org/10.7322/jhgd.124092

10.Pereira SG, Carvalho AJA, Escarce AG, Alves JMM, Goulart LMHF, Lemos SMA. Hearing trial in child education: association with health determinants. Disturb Comun 2019;31:285-96. http://dx.doi.org/10.23925/2176-2724.2019v31i2p285-296

11. Moher D, Shamseer L, Clarke M, Ghersi D, Liberati A, Petticrewet $M$, et al. Preferred reporting items for systematic review and metaanalysis protocols (PRISMA-P) 2015 statement. Syst Rev 2015;4:1. https://doi.org/10.1186/2046-4053-4-1

12.Pithon MM, Sant'anna LIDA, Baião FCS, Santos RL, Coqueiro RS, Maia LC. Assessment of the effectiveness of mouthwashes in reducing 
cariogenic biofilm in orthodontic patients: a systematic review. J Dent 2015;43:297-308. https://doi.org/10.1016/j.jdent.2014.12.010

13.Pereira MB, Befi-Lopes DM, Samelli AG. Association between audiological profile and primary language impairment in children. International. Int $\mathrm{J}$ Pediatr Otorhinolaryngol 2015;79:53-7. https://doi.org/10.1016/j.ijporl.2014.11.003

14.Lopes DMB, Cáceres AM, Esteves L. Perfil linguístico de crianças com alteração específica de linguagem. Rev Soc Bras Fonoaudiol 2012;17:274-8. https://doi.org/10.1590/S1516-80342012000300007 15.Prates LPCS, Martins VO. Distúrbios da fala e da linguagem na infância. Rev Med Minas Gerais 2011;21:5460. https://ftp.medicina.ufmg.br/ped/Arquivos/2013/disturbiofalaeim agem8periodo 2108 2013.pdf

16. Hage SRV, Faiad LNV. Perfil de pacientes com alteração de linguagem atendidos na clínica de diagnóstico dos distúrbios da comunicação - Universidade de São Paulo - campus Bauru. Rev CEFAC 2005; 7:433-40.

https://www.redalyc.org/pdf/1693/169320507006.pdf

17.Barros PML, Oliveira PN. Perfil dos pacientes atendidos no setor de Fonoaudiologia de um serviço público de Recife - PE. Rev CEFAC 2010;12:128-33. https://doi.org/10.1590/S1516-

\section{3}

18.Guida HL, Diniz TH. Audiological Profile in Children Aging from 5 to 10 Years. Int Arch Otorhinolaryngol 2008;12:224-9. http://arquivosdeorl.org.br/additional/acervo eng.asp?id $=518$

19.Araújo SA, Moura JR, Camargo LA, Alves W. Avaliação auditiva em escolares. Rev Bras Otorrinolaringol 2002;68:263-6. https://doi.org/10.1590/S0034-72992002000200017

20.Oliveira LN, Goulart BNG, Chiari BM. Distúrbios de linguagem associados à surdez. Rev Bras Cres Desenvolv Hum 2013;23:41-5. https://doi.org/10.7322/jhgd.50389

21. Francesco RC, Barros VB, Ramos R. Otite média com efusão em crianças menores de um ano. Rev Paul Pediatr 2016;34:148-53.

http://dx.doi.org/10.1016/j.rppede.2016.01.003

22.Balbani APS, Montovani JC. Impacto das otites médias na aquisição da linguagem em crianças. J Pediatr 2003;79:391-6. https://doi.org/10.1590/S0021-75572003000500005

23.Dimer TV, Wender MH, Reis HG, Taguchi CK. Timpanometria em pacientes com otite média secretora submetidos a tratamento com corticosteróides e manobras de insuflação. Sci Med (Porto Alegre) 2005; 15:249-53.

https://revistaseletronicas.pucrs.br/ojs/index.php/scientiamedica/arti cle/download/1575/1178/

24.Etges $C L$, Reis MCP, Menegotto IH, Sleifer $P$, Soldera CLC. Acoustic immitance and auditory processing screening findings in school children. Rev CEFAC 2012;14:1098-107. https://doi.org/10.1590/S1516-18462012005000028 
25.Santos MFC, Bragato GR, Martins PMF, Dias AB. Auditory assessment in the school-age children. Rev CEFAC 2009;11:64453. https://doi.org/10.1590/S1516-18462009000800013

26. Lindau TA, Delecrode CR, Cardoso ACV. Tympanometric findings in a group of students. Rev CEFAC 2013;15:1453-60. https://doi.org/10.1590/S1516-18462013005000036

27.Leite RFP, Santos MSA, Ribeiro EM, Pessoa ALS, Lewis DR, Giacheti $\mathrm{CM}$, et al. Triagem auditiva de crianças com síndrome congênita pelo vírus Zika atendidas em Fortaleza, Ceará, 2016. Epidemiol Serv Saude 2018;27:e2017553. https://dx.doi.org/10.5123/s167949742018000400002

28.Vasconcelos RM, Monte MO, Aragão VMF, Silva BTF. Alterações auditivas em crianças de 7 a 9 anos de idade de uma escola pública de ensino fundamental em São Luís, Maranhão. Prom Saúde 2007;20:155-60. https://www.redalyc.org/articulo. oa?id=40820304 29.Parra GFA, Carvallo RMM, Nakagawa L. Reflexos Acústicos Eliciados com Sonda de 678 e $1.000 \mathrm{~Hz}$ em Adultos sem Queixa Auditiva. Int Arch Otorhinolaryngol 2005;9:27-36. http://www.arquivosdeorl.org.br/conteudo/acervo port.asp? Id =300 30.César AM, Maksud SS. Caracterização da demanda de fonoaudiologia no serviço público municipal de Ribeirão das Neves MG. Rev CEFAC 2007;9:133-8. https://doi.org/10.1590/S1516$\underline{18462007000100017}$ 